\title{
Primary Extra-Osseous Ewing Sarcoma of the Thyroid: A Case Report and Review of the Literature
}

\author{
Amanda H. Seipel ${ }^{1} \cdot$ Hiba Mechahougui ${ }^{2} \cdot$ Nicolas Mach $^{2} \cdot$ Frédéric Triponez $^{3} \cdot$ William C. Faquin $^{4} \cdot$ Claudio De Vito $^{1}$ (i)
}

Received: 2 June 2021 / Accepted: 14 July 2021 / Published online: 26 July 2021

(c) The Author(s) 2021

\begin{abstract}
Extra-osseous Ewing sarcoma (ES) is a rare and aggressive malignant tumor found in a variety of organs. Primary ES of the thyroid is exceedingly rare and few cases have been documented to date. We describe the case of a 54-year old woman with a history of breast carcinoma in whom a unique hypermetabolic left thyroid nodule was identified during a follow-up PET-CT scan. An ultrasound examination showed a hypoechogenic nodule of $3.7 \mathrm{~cm}$. A cytological diagnosis of poorly differentiated thyroid carcinoma was made, and a total thyroidectomy was performed. The surgical specimen revealed a poorly differentiated neoplasm composed of medium-sized cells with scant cytoplasm, expressing pancytokeratin, CD99 and NKX2.2 but lacking p63 and p40 expression. Molecular analysis revealed a EWSRI-FLII fusion transcript supporting the diagnosis of a primary extra-osseous ES of the thyroid. The patient received adjuvant chemotherapy and has no evidence of recurrent disease.
\end{abstract}

Keywords Thyroid gland $\cdot$ Extra-osseous Ewing sarcoma $\cdot$ Cyto-histological correlation $\cdot$ Molecular analysis

\section{Introduction}

Thyroid cancers encompass a wide range of tumors, from indolent cases with low mortality to extremely aggressive malignancies with short overall survival. Proper diagnostic work-up and classification are crucial to tailor patient treatment. Well-differentiated thyroid carcinoma such as papillary, follicular and Hürthle cell carcinoma account for more than $95 \%$ of all cases, while other subtypes are less frequently seen [1]. Mesenchymal thyroid neoplasms are exceedingly rare among which Ewing sarcoma (ES) represents only $2 \%$ [2]. ES is a member of the so-called "small round blue cell" tumors, most commonly arising in bone and

Claudio De Vito

claudio.devito@hcuge.ch

1 Department of Clinical Pathology, Geneva University Hospital, Geneva, Switzerland

2 Department of Clinical Oncology, Geneva University Hospital, Geneva, Switzerland

3 Department of Thoracic and Endocrine Surgery, Geneva University Hospitals and Faculty of Medicine, University of Geneva, Geneva, Switzerland

4 Department of Pathology, Massachusetts General Hospital and Harvard Medical School, Boston, MA 02114, USA most frequently found in children and adolescents. Extraosseous Ewing sarcoma (EES) is less frequent and can occur at a wide variety of anatomic [3]. Several recent reports have described an adamantinoma-like Ewing sarcoma (ALES) arising in the thyroid gland, based on the identification of EWSR 1 rearrangement in neoplasms showing the classic ES morphology with epithelial differentiation (keratin and p63 or p40 expression) [4-8], and carcinoma of the thyroid with Ewing family tumor elements (CEFTEs) when associated with papillary thyroid carcinoma [9-12]. Here we present the case of a patient diagnosed with EES located in the left thyroid lobe and a review of the current literature.

\section{Case}

\section{Clinical History}

A 54-year old woman presented with a $3.7 \mathrm{~cm}$ nodule in the left thyroid lobe during a routine PET-scan follow-up for a triple negative breast carcinoma, without BRCA-1 or BRCA-2 mutation, treated with neoadjuvant chemotherapy, surgery, and radiotherapy. Physical examination revealed an asymmetric thyroid gland with a firm nodule of the left thyroid lobe, without any palpable lymphadenopathy. 
Ultrasound imaging revealed a hypoechogenic and hypervascularized nodule measuring $3.7 \times 3.1 \times 2.1 \mathrm{~cm}$ without calcifications which was classified radiologically as TiRADS 4 .

An US-guided fine needle aspiration (FNA) was performed which showed a monomorphic proliferation of dyscohesive cells isolated and in groups, with scant cytoplasm and distinct small nucleoli (Fig. 1A). The cells stained diffusely for pancytokeratin (Fig. 1B), and scattered cells stained for TTF-1 (Fig. 1C) and thyroglobulin (data not shown). GATA-3, calcitonin, mammaglobin, estrogen and progesterone receptors were negative (data not shown). Based on the cytomorphology and immunocytochemical profile a diagnosis of poorly differentiated thyroid carcinoma (PDTC) was made, and the patient underwent a total thyroidectomy.

The surgical specimen of the left thyroid lobe showed a poorly delineated cream-colored nodule with hemorrhagic zones, measuring $4 \mathrm{~cm}$, occupying the superior and medial part of the thyroid, which was extensively sampled. The right lobe contained a small, sclerotic nodule.

Histological analysis showed a solid cellular proliferation separated by fibrous bands, composed of small to medium sized cells with scant cytoplasm and an enlarged nucleus. Chromatin was evenly distributed and nucleoli were inconspicuous (Fig. 2A). Mitoses were scarce. Multifocal vascular invasion was observed, without any evidence of lymphatic or perineural invasion. The tumor was limited to the thyroid gland but focally in contact with the surgical margins. Immunohistochemistry showed diffuse expression for pancytokeratin, epithelial membrane antigen (EMA), CD117,
CD99 (Fig. 2B) and NKX2.2. The cells were negative for PAX-8, thyroglobulin, CEA, calcitonin, p63, p40 (Fig. 2C), chromogranin, synaptophysin, hormonal receptors (ER, PR), GATA3, mammaglobin and BRST2. P53 showed a wild type expression. INI-1 and BRG-1 expression was conserved. Scattered non-neoplastic thyrocytes expressing TTF-1 were observed entrapped within the tumor. The surrounding thyroid parenchyma showed mild chronic thyroiditis.

Based on the histomorphology and immunohistochemical profile, an RNA-based fusion panel (Illumina TruSight Fusion panel, Illumina, San Diego, CA) was performed and identified an EWSRI-FLII fusion transcript, validated by a EWSRI FISH break apart probe (Fig. 2D) supporting the diagnosis of EES. An additional custom NGS targeted panel (including more than 400 genes) did not identify any gene mutation. Since PET/CT did not reveal involvement of other anatomic sites, a diagnosis a primary EES of the thyroid gland was made. Based upon the overall pathologic and clinical findings, the multidisciplinary institutional tumor board panel recommended adjuvant chemotherapy.

The patient then received four cycles of adjuvant polychemotherapy with VIDE (Vincristine, Ifosfamide, Doxorubicin, Etoposide) and Peg G-CSF.

Adequate cardiac function was confirmed by cardiac ultrasound as part of the pre-treatment protocol and because the patient had already received $360 \mathrm{mg} / \mathrm{m}^{2}$ of Epirubicine in 2016 for the multimodality treatment of a triple negative breast cancer. Chemotherapy was complicated by grade 3 asthenia, and grade 4 neutropenia, anemia, and thrombocytopenia requiring a $20 \%$ dose reduction for the last two
Fig. 1 Cytological findings in the FNA. A Monomorphic proliferation of dyscohesive cells, with scant cytoplasm and visible nucleoli, PAP stain $(\times$ 100). B Keratin immunocytochemistry $(\times 100)$. C TTF1 immunocytochemistry showing nuclear expression in a subset of cells $(\times 100)$
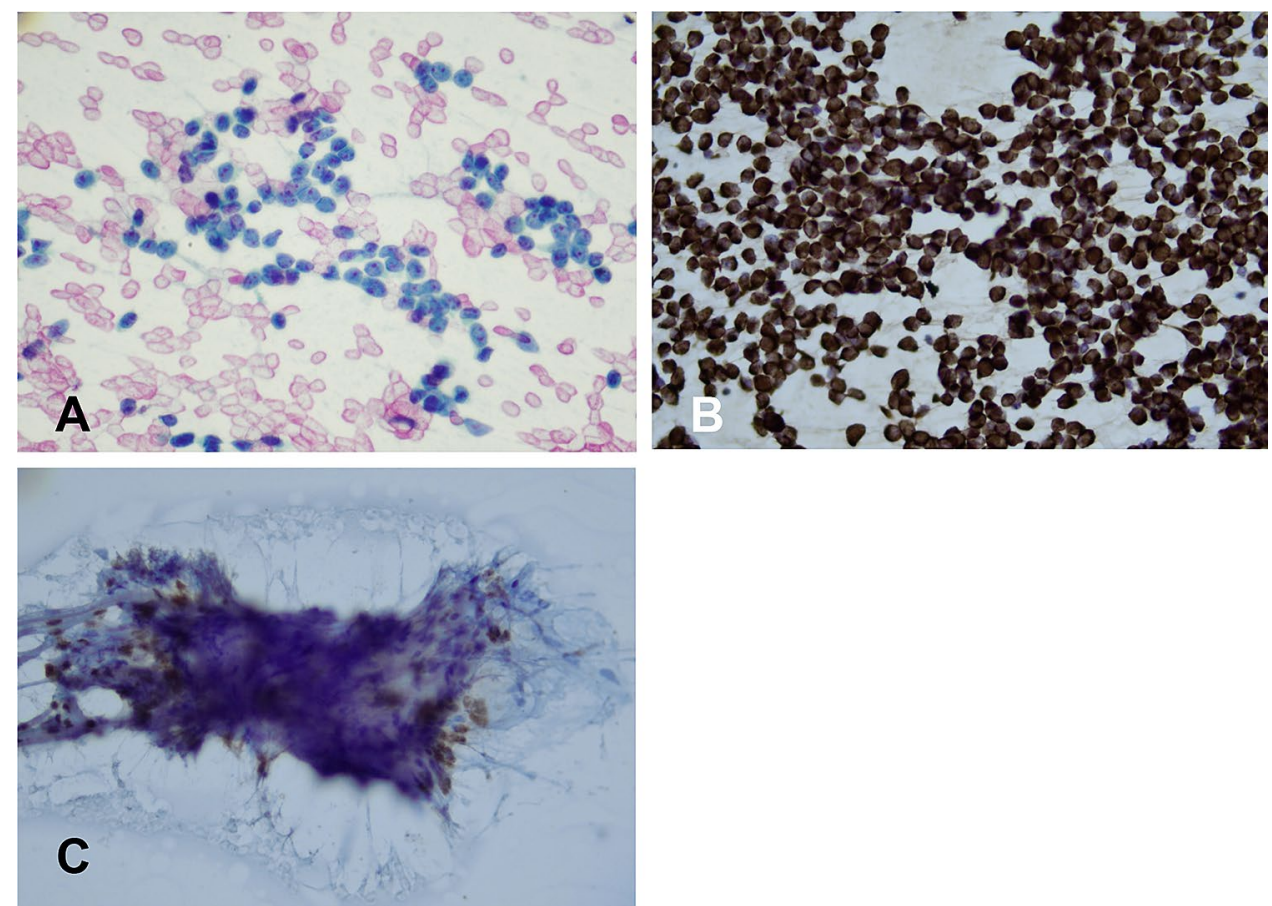
Fig. 2 Histological, immunohistochemical and FISH findings in the surgical specimen. A Neoplasm composed of small to medium sized cells with scant cytoplasm and enlarged nucleus with evenly distributed chromatin and inconspicuous nucleoli (H\&E*). B Diffuse CD99 expression $(\times 100)$. C Absence of p40 expression $(\times 100), \mathbf{D}$ FISH analysis showing EWSR 1 gene rearrangement using a break apart probe. "Hematoxylin and eosin
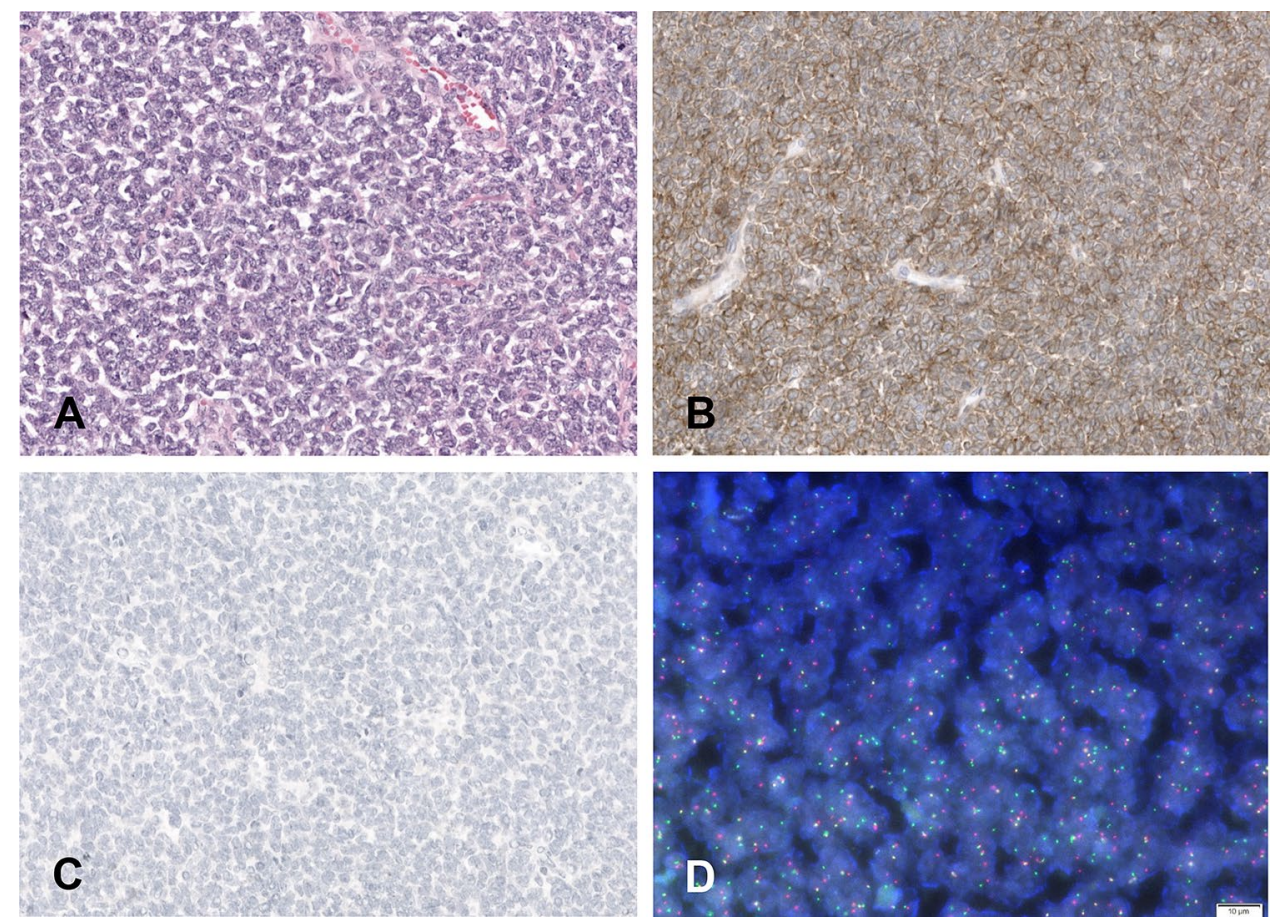

cycles. Treatment was completed with adjuvant radiotherapy (total dose $45 \mathrm{~Gy}$ delivered in 25 sessions). As this patient presented with two rare and aggressive neoplasms, triple negative breast cancers and a sarcoma developed within the field of her breast cancer radiotherapy, genetic predisposition such as Li-Fraumeni syndrome was suspected. Genetic analysis of eight genes: TP53, PTEN, STK11, PALB2, ATM, CHEK 2, did not reveal any anomaly.

Fifteen months after surgery, the patient is in remission with no local recurrence no distant metastases.

\section{Discussion}

Primary ES family tumors tend to occur most commonly in bone, primarily in adolescents and young adults, and is characterized by chromosomal rearrangements involving the EWSRI gene [3]. More recently EES termed ALES has been increasingly recognized in the head and neck region including salivary gland and thyroid [13], and also carcinoma of the thyroid with CEFTEs [9]. Although ES can express epithelial markers such as pankeratin [14], p63 and/ or p40 were reported only in ALES [13]. In our case, the absence of $\mathrm{p} 40$ and $\mathrm{p} 63$ expression and the lack of papillary thyroid carcinoma foci associated with the tumor support the classification of EES over ALES or CEFTE.

The pre-operative FNA diagnosis remains challenging (Table 1). As reported by Kabata et al. [15], a correct FNA diagnosis was not achieved in any case. More recently one report [16] describes a successful interpretation by FNA.
It is interesting to note that the few cases that were able to correctly diagnose the tumor were done in association with a core needle biopsy [15, 16] or excision biopsy [4] (Table 1).

As in our case, a potential pitfall in the interpretation of cytological specimens is the diffuse expression of keratin, which may lead to the misclassification of the cancer as PDTC. This is especially true when entrapped benign, TTF-1 positive thyrocytes are present in the FNA sample as encountered in our case, and similarly as described by Chikeka et al. [17], or when p40 or p63 are only expressed focally $[6,8]$, or are absent.

The differential diagnosis encompasses a broad range of neoplasms including metastatic basaloid squamous cell carcinoma, which expresses keratin, p63 and p40, and medullary thyroid carcinoma which can be confirmed by testing for calcitonin and CEA expression. In the setting of a calcitonin-negative tumor, other neuroendocrine entities of the thyroid gland including intrathyroidal paraganglioma should be considered. Intrathyroidal thymic carcinoma based on the immunohistochemical profile (positivity for keratin, EMA and CD117) should also be excluded.

Given the high-grade nature of the tumor and unusual immunoprofile and histomorphology, a thyroid gland metastasis should also be considered. In our case, a metastasis from the patient's known breast cancer was excluded using immunohistochemical markers for GATA-3, mammaglobin, and BRST2. This clinical presentation of EES is reminiscent of the case reported by Maldi et al. [6].

ES is an aggressive disease with a $70 \%$ overall 5-year relative survival rate [3]. However, the difference in 


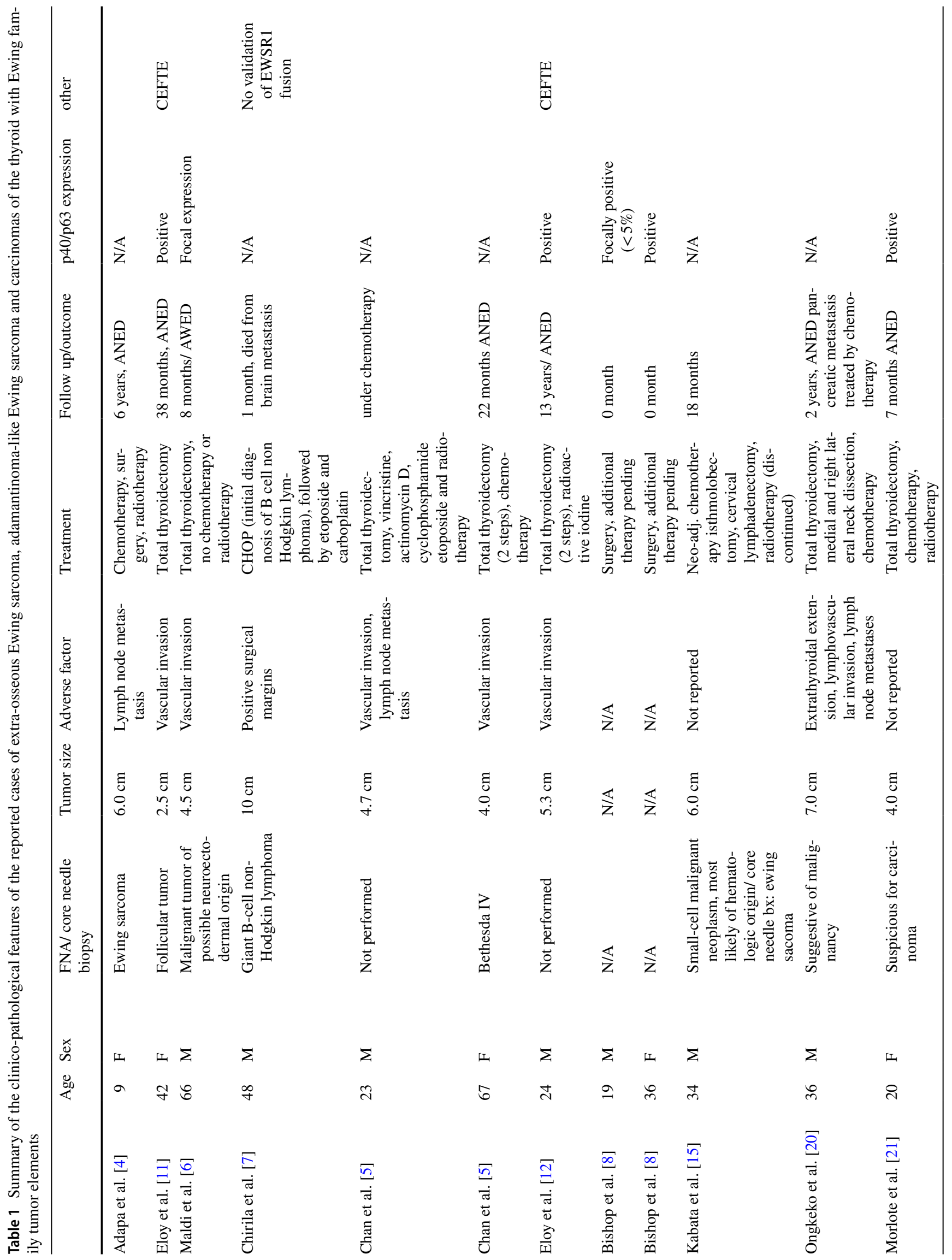


prognosis between EES and ES is still controversial. A report from the Children's Oncology Group analyzing a cohort of two clinical trials including 213 extraskeletal ES and 826 skeletal ES showed that EES were associated with a slightly better event-free survival [18]. In contrast, Lynch et al. [19] did not find any impact on survival in the adult population. Most patients with thyroid involvement presented with angio-lymphatic invasion and a small subset with lymph node metastasis (Table 1). The majority of patients received adjuvant chemotherapy and all showed no evidence of disease in the clinical follow-up except for one patient [20]. However, it is important to emphasize that the clinical follow-up for most patients was limited, and therefore any conclusions about the long term patient prognosis is uncertain.

In conclusion, correct pre-operative diagnosis of extraosseous ES remains challenging. Pathologists, including those specializing in cytopathology, should be aware that ES should be considered in the differential diagnosis of poorly differentiated tumors of the thyroid, including those with diffuse keratin expression and focal expression of $\mathrm{p} 40 /$ p63. For such cases, CD99 and NKX2.2 immunostaining or molecular analysis with NGS or FISH can be helpful to properly classify the tumor which is of crucial importance for proper patient management.

Authors contribution AHS and CDV wrote the manuscript, AHS, CDV and WCF provided the pathological pictures, HM, NM and FT provided clinical information, CDV supervised the manuscript. All the authors reviewed and edited the manuscript.

Funding Open Access funding provided by Université de Genève. No funds, grants, or other support was received.

Data and material availability Not applicable.

Code availability Not applicable.

\section{Declarations}

Conflict of interest The authors declare that there is no conflict of interest.

Ethics Approval Not required.

Consent to Participate Not applicable.

Consent for Publication Written consent from the patient.

Open Access This article is licensed under a Creative Commons Attribution 4.0 International License, which permits use, sharing, adaptation, distribution and reproduction in any medium or format, as long as you give appropriate credit to the original author(s) and the source, provide a link to the Creative Commons licence, and indicate if changes were made. The images or other third party material in this article are included in the article's Creative Commons licence, unless indicated 
otherwise in a credit line to the material. If material is not included in the article's Creative Commons licence and your intended use is not permitted by statutory regulation or exceeds the permitted use, you will need to obtain permission directly from the copyright holder. To view a copy of this licence, visit http://creativecommons.org/licenses/by/4.0/.

\section{References}

1. Haddad RI, Nasr C, Bischoff L, et al. NCCN Guidelines insights: thyroid carcinoma, version 2.2018. J Natl Compr Canc Netw. 2018;16:1429-40.

2. Gubbi S, Thakur S, Avadhanula S, et al. Comprehensive guidance on the diagnosis and management of primary mesenchymal tumours of the thyroid gland. Lancet Oncol. 2020;21:e528-37.

3. Riggi N, Suvà ML, Stamenkovic I. Ewing's sarcoma. N Engl J Med. 2021;384:154-64.

4. Adapa P, Chung T-W, Popek EJ, Hunter JV. Extraosseous Ewing sarcoma of the thyroid gland. Pediatr Radiol. 2009;39:1365-8.

5. Chan JM, Bilodeau E, Celin S, et al. Ewing sarcoma of the thyroid: report of 2 cases and review of the literature. Head Neck. 2013;35:E346-50.

6. Maldi E, Monga G, Rossi D, et al. Extra-osseous Ewing sarcoma of the thyroid gland mimicking lymphoma recurrence: a case report. Pathol Res Pract. 2012;208:356-9.

7. Chirila M, Muresan M, Ciuleanu E, Cosgarea M. Extraosseous Ewing sarcoma and peripheral primitive neuroectodermal tumor of the thyroid gland: case report and review. Ear Nose Throat J. 2013;92:E3-6.

8. Bishop JA, Alaggio R, Zhang L, et al. Adamantinoma-like Ewing family tumors of the head and neck: a pitfall in the differential diagnosis of basaloid and myoepithelial carcinomas. Am J Surg Pathol. 2015;39:1267-74.

9. Oliveira G, Polónia A, Cameselle-Teijeiro JM, et al. EWSR1 rearrangement is a frequent event in papillary thyroid carcinoma and in carcinoma of the thyroid with Ewing family tumor elements (CEFTE). Virchows Arch. 2017;470:517-25.

10. Taccogna S, Guglielmi R, Persichetti A, et al. Carcinomas of the thyroid with Ewing family tumor elements (CEFTEs): a diagnostic challenge before surgery. Head Neck Pathol. 2021;15:254-61.
11. Eloy C, Oliveira M, Vieira J, et al. Carcinoma of the thyroid with Ewing family tumor elements and favorable prognosis: report of a second case. Int J Surg Pathol. 2014;22:260-5.

12. Eloy C, Cameselle-Teijeiro J, Vieira J, et al. Carcinoma of the thyroid with Ewing/PNET family tumor elements: a tumor of unknown histogenesis. Int J Surg Pathol. 2014;22:579-81.

13. Rooper LM, Bishop JA. Soft tissue special issue: adamantinomalike Ewing sarcoma of the head and neck: a practical review of a challenging emerging entity. Head Neck Pathol. 2020;14:59-69.

14. Srivastava A, Rosenberg AE, Selig M, et al. Keratin-positive Ewing's sarcoma: an ultrastructural study of 12 cases. Int J Surg Pathol. 2005;13:43-50.

15. Kabata P, Kaniuka-Jakubowska S, Kabata W, et al. Primary Ewing sarcoma of the thyroid-eight cases in a decade: a case report and literature review. Front Endocrinol (Lausanne). 2017;8:257.

16. Jones R, Maleki Z. Adamantinoma-like Ewing sarcoma of the thyroid. Diagn Cytopathol. 2020;48:E4-6.

17. Chikeka I, Sung S, Tiscornia-Wasserman P, Cimic A. Adamantinoma-like Ewing sarcoma of the thyroid gland: cytomorphologic, phenotypic and molecular features. Diagn Cytopathol. 2020;48:1107-10.

18. Cash T, Mcllvaine E, Krailo MD, et al. Comparison of clinical features and outcomes in patients with extraskeletal versus skeletal localized Ewing sarcoma: a report from the Children's Oncology Group. Pediatr Blood Cancer. 2016;63:1771-9.

19. Lynch AD, Gani F, Meyer CF, et al. Extraskeletal versus skeletal Ewing sarcoma in the adult population: controversies in care. Surg Oncol. 2018;27:373-9.

20. Ongkeko M, Zeck J, de Brito P. Molecular testing uncovers an adamantinoma-like Ewing family of tumors in the thyroid: case report and review of literature. AJSP Rev Rep. 2018;23:8-12.

21. Morlote A, Harada S, Lindeman B, Stevens SM. Adamantinomalike Ewing sarcoma of thyroid: a case report and review of the literature. Head Neck Pathol. 2019;13:618-23.

Publisher's Note Springer Nature remains neutral with regard to jurisdictional claims in published maps and institutional affiliations. 